\title{
Desktop Load/Deflection Measurement
}

\author{
Devin R. Berg
}

11 May 2011

\begin{abstract}
In order to achieve a low-cost solution for the collection of load/deflection data, a simple device based upon a balance like mechanism and two variable measurements was designed and tested. The device uses a linear variable differential transformer and a potentiometer to determine the load applied to the test specimen and the extension that the specimen exhibits under such a load. Test samples of known characteristics were used in order to provide a basis for gaging the performance of the device against well established measurement systems. It was found that the slope of the load/deflection curve obtained with this device was within $10 \%$ of the accepted data. This performance is considered reasonable for a device of this nature and several areas for improvement are discussed.
\end{abstract}

\section{Introduction}

The quantification of material properties is an essential consideration in many areas of engineering. For example, more accurate estimates of material performance permits greater confidence in a proposed solution. Further, greater understanding of a biomaterial makes it possible to predict the behavior of biological systems. Conventional systems for characterizing material properties (shown in Figure 1) have been widely accepted as a standard of measurement; however, their high price make them inaccessible to many small businesses or research labs that would benefit from such a system. 


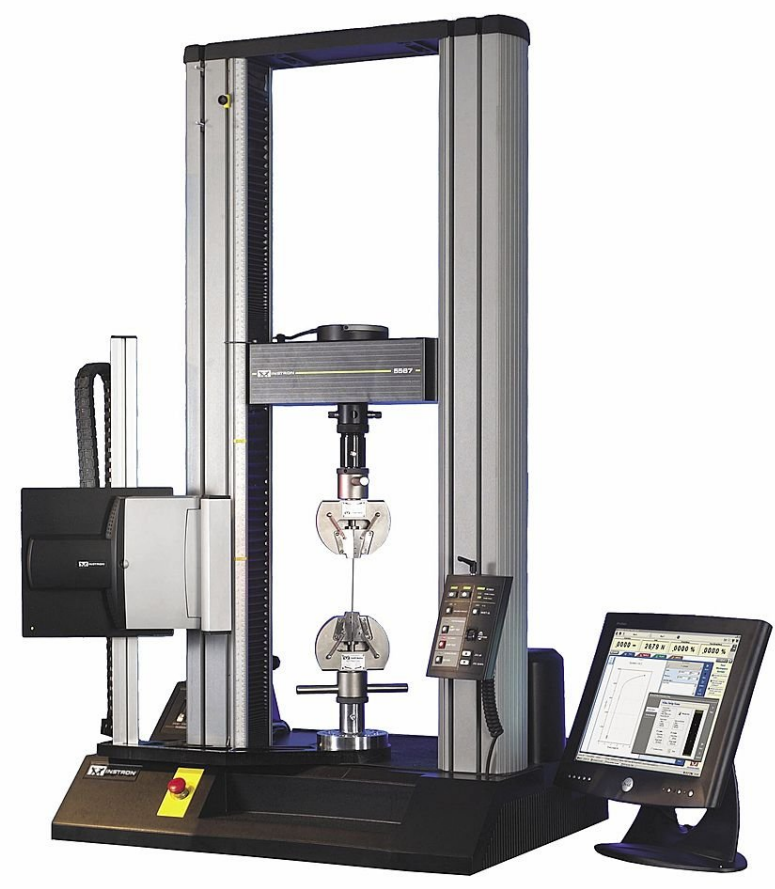

Figure 1: Image of an Instron tension tester [1].

The basic concept for a simple uniaxial testing apparatus is the ability to apply a variable load to a test specimen and record the corresponding deflection of the specimen. These load and deflection data points can then be converted into stress and strain assuming that the geometry of the specimen is known. If a sufficient number of stress and strain data points are obtained, the stress-strain curve (Figure 2) can be produced and thus basic information on the material strength or other material specifications can be determined. Ideally, the apparatus should be capable of applying a continuously increasing load for a more accurate curve that doesn't require interpolation. 


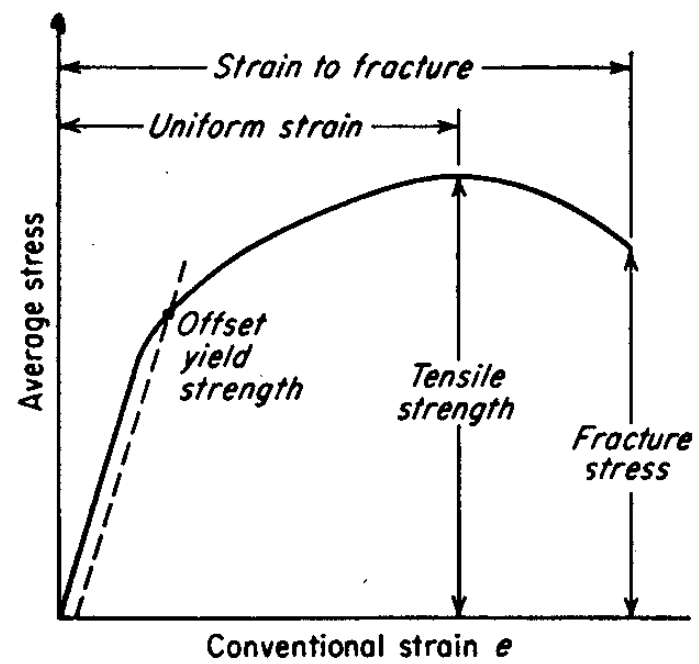

Figure 2: Example stress-strain curve [2].

In order to determine the performance of the device, load and deflection data will be collected using sample test specimens which exhibit large strain behavior. This data will be compared against similar data that was collected using a standard tension testing machine. To quantify this evaluation, the slopes of the load/deflection plots will be compared with a target goal of having $<10 \%$ deviation between the standard test data and the data collected using this device.

\section{Design}

To meet the aforementioned specifications, a design was developed that is both simple and producible using available components. In order to achieve linearly scaling force applied to the sample, a balance mechanism with a variable moment arm on the load side was chosen. This permitted the linear distance between the load and the fulcrum to be increased thus increasing the mechanical advantage of the system and the force applied to the sample, as illustrated in Figure 3. This relationship is shown in Equation 1 where $F$ is the force applied to the sample, $P$ is the static load, $\theta$ is the angular orientation of the moment arm, and $L_{s}$ and $L_{p}$ are the distances between the fulcrum and the sample and load, respectively.

$$
F=\frac{L_{p}}{L_{s}} \cdot \frac{\cos \theta}{\cos \frac{\theta}{2}} \cdot P
$$

From this equation, it can be seen that the force applied to the sample is equal to the static load scaled by the ratio of the moment arms. Additionally there is a correction for the angular orientation of the beam; however, this correction is nearly unity for small angles $\left(<20^{\circ}\right)$. Further, since $L_{s}$ is fixed in this design the applied force is proportional to the variable load moment arm, $L_{p}$. 


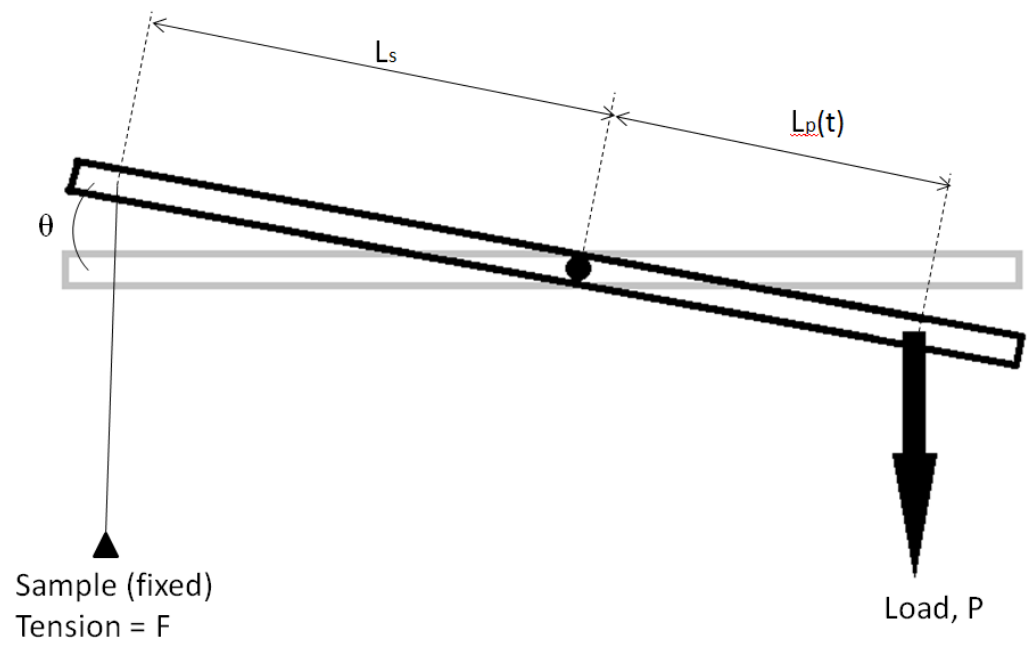

Figure 3: Governing geometric relationships for sample force and extension.

The second piece of information that is necessary for load/deflection data is the extension of the sample. By again considering the geometry shown in Figure 3 the extension of the sample, $d l$, can be found as presented in Equation 2.

$$
d l=L_{s} \cdot \sqrt{2(1-\cos \theta)}
$$

Recalling that $L_{s}$ is a constant value, the only variable remaining in this equation is the angular orientation of the moment arm, $\theta$. Taking Equations 1 and 2 into consideration together, it is apparent that there are only two unknown values, $L_{p}$ and $\theta$. Therefore, these two value must be measured using the device in order to arrive at the desired parameters, load and extension. Thus a design to achieve this was developed and modeled using the software package Pro/Engineer as shown in Figure 4. The system consists of a passive structural assembly as well as several active components for manipulation and measurement. 


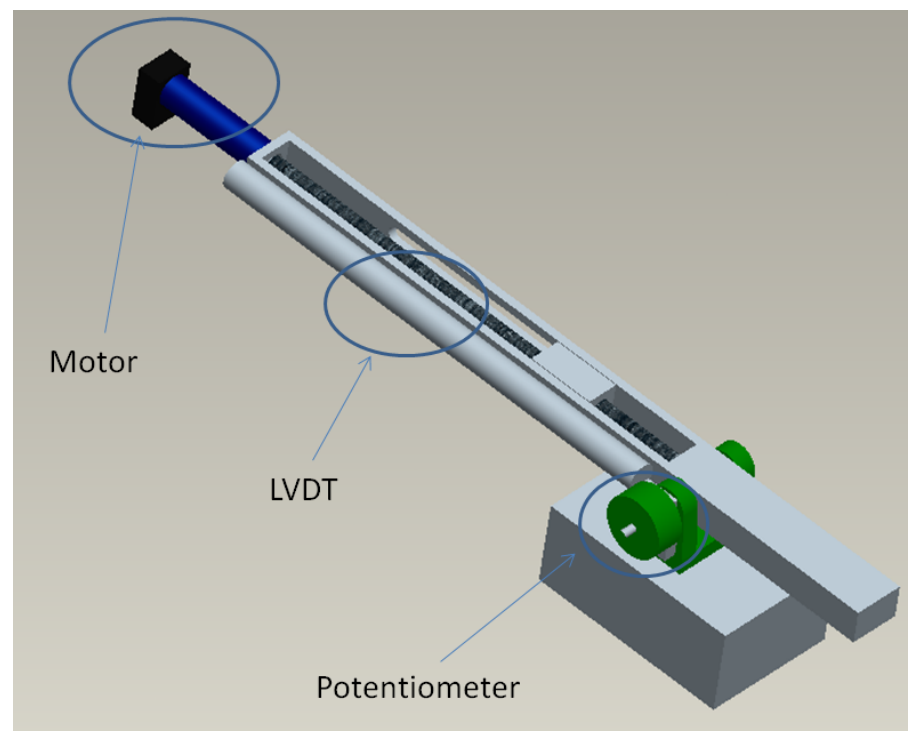

Figure 4: Pro/Engineer model of the proposed design with major components labeled.

The active components labeled in Figure 4 include the motor, the linear variable differential transformer (LVDT), and the potentiometer. These components were selected for their measurement capability as well as their availability. The motor assembly consists of a DC motor and a 415:1 reducing planetary gearhead (23/1, Minimotor SA, Faulhaber) which provides low-speed, high-torque rotary motion. The LVDT (3000HR-DC, Schaevitz) measures linear displacement using three solenoid coils and a mobile ferromagnetic core by measuring signal variation between the two outer coils. This method enables the LVDT to have a theoretically infinite resolution [3]. The final active component is the rotary position sensing potentiometer (CPP-45B, Midori America Corp.). The rotary potentiometer has a sliding contact that allows the device to operate as a variable voltage divider. Thus a constant, known voltage is input and the output is measured as a variable voltage signal that is some fraction of the total input voltage amplitude. For the potentiometer used here, the linearity of the device is $\pm 0.3 \%$ and the repeatability is $\pm 0.003 \%$ $[4]$.

The passive structure for this device was produced in the machine shop and is shown in assembled form in Figure 5. In this image, a test specimen is hanging from the sample mounting point but is not secured on the opposing end. 


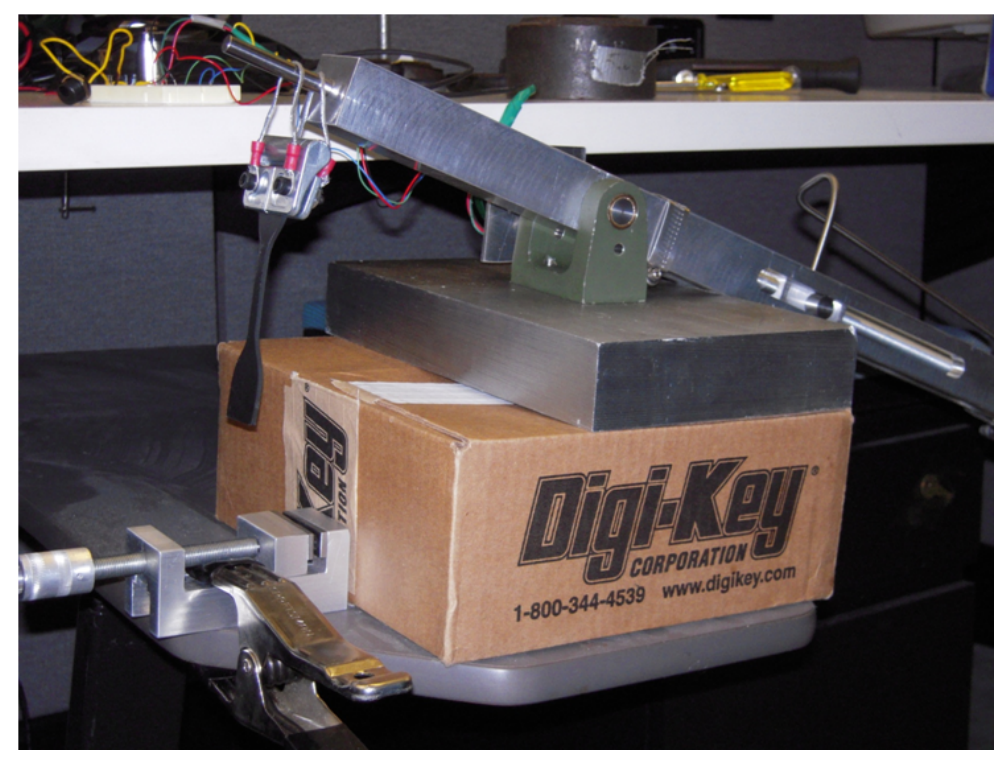

Figure 5: Assembled load/deflection measurement device.

\section{Experimental Results}

In order to determine whether or not the device performs in an acceptable manner, it was necessary to measure the load/deflection response for a material of known characteristics. Thus tensile test specimens of a rubber compounds were obtained. The load/deflection response of the specimens was known based upon testing performed using a MTS tension testing machine, considered to be an accepted standard in the field. For the purposed of this testing, deviations of up to $10 \%$ between the measured and expected slope of the load/deflection plot would be considered the target range.

Prior to testing, the voltage readings from the LVDT and the potentiometer were mapped respectively to linear displacement and angular readings assuming linearity. Further, due to limitations on the available equipment, measurement readings were taken at discrete points rather than continuously as the distance between the static load and the fulcrum was increased. The experimental setup is shown in Figure 6 where the static load is applied and the test specimen is under tension (left) as well as an example voltage reading being taken from the potentiometer output (right). 

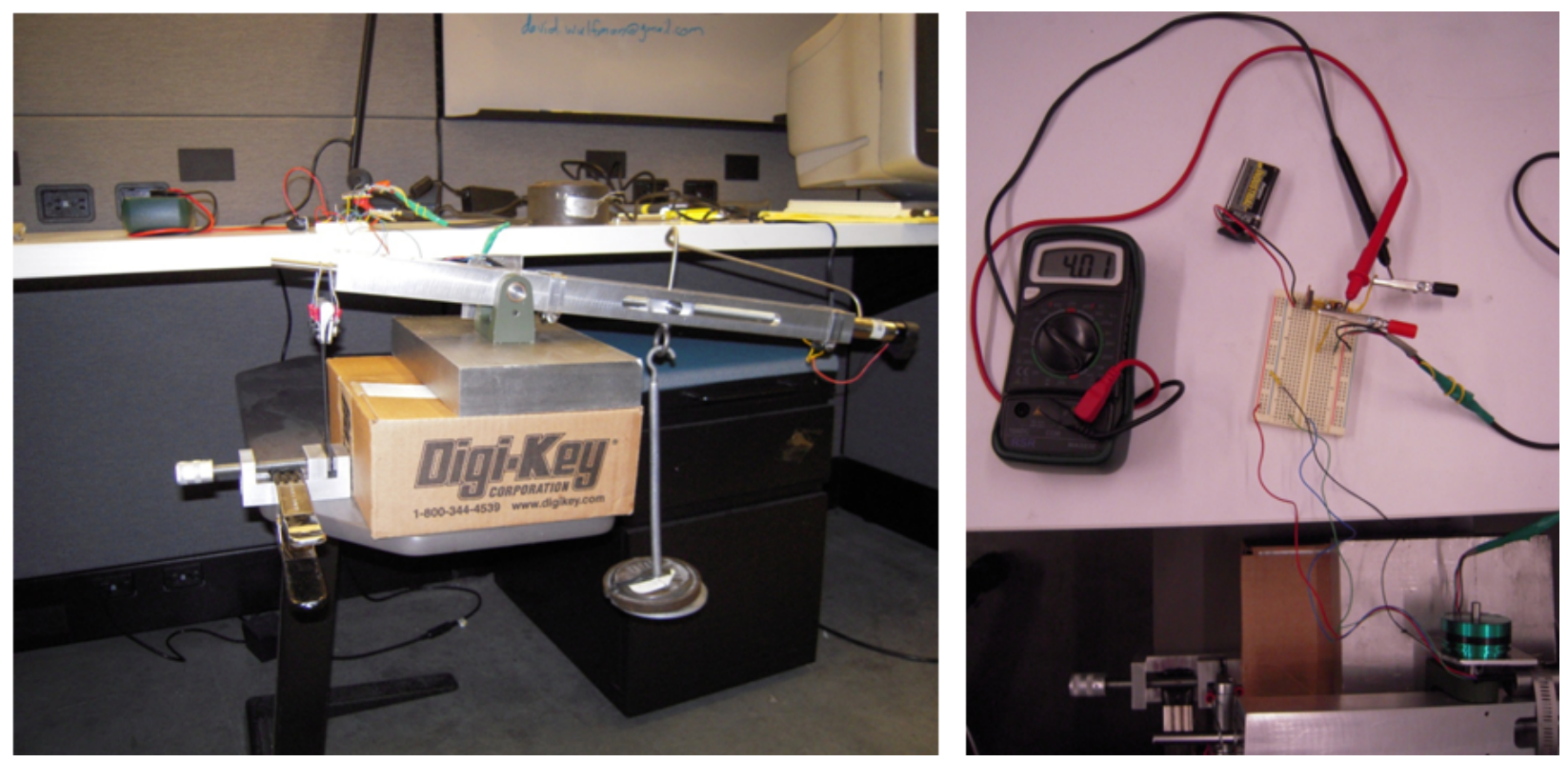

Figure 6: Experimental evaluation of the device with (left) the test specimen placed under tensile load and (right) an example of a voltage reading being taken from the potentiometer.

For this experimental evaluation, three separate samples were tested by varying the load moment arm through its full range of approximately 5.5 inches. These three data sets are shown plotted with the three data sets collected on the MTS tension testing machine in Figure 7 . In this plot, the top data sets are those collected from the proposed device. By plotting a linear trend-line for each of the data sets it was found that the slope of this line ranged between 0.65 and $0.66 \frac{\mathrm{N}}{\mathrm{mm}}$ with only slight variations in the overall offset of the force measurements. In addition, plotted below are the data sets collected from the MTS machine where the line slopes ranged from 0.69 to $0.75 \frac{\mathrm{N}}{\mathrm{mm}}$. Thus for the MTS machine there was a slightly larger deviation between data sets than there was for the proposed device. Further, by comparing between the three test averages of both the MTS and the proposed device, it was found that the proposed device measured slopes approximately 9.2 $\%$ lower than for the MTS machine. This is a large variation but remains within the specification set forward at the start of the project. 


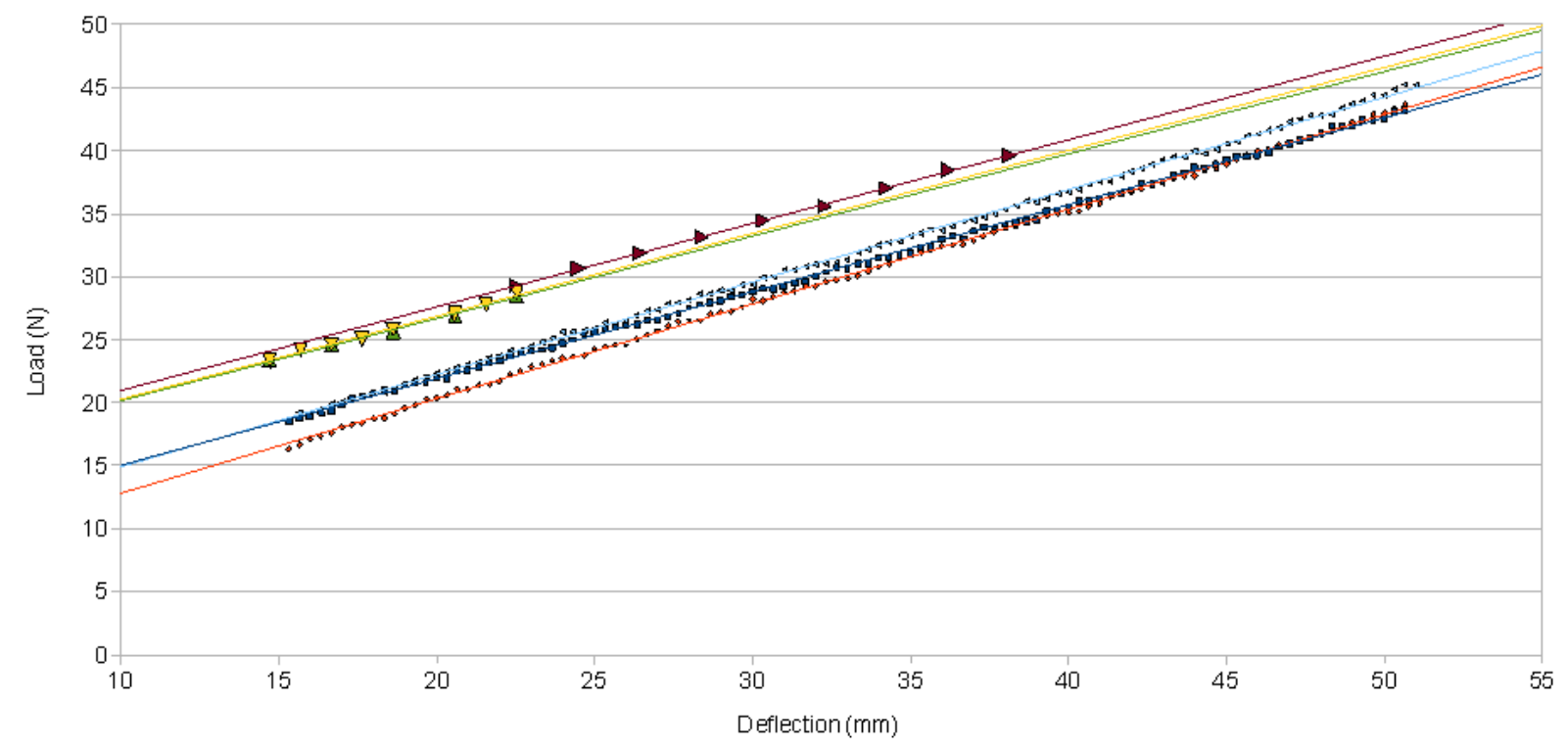

Figure 7: Experimental results collected using the proposed device along with data collected using the MTS tension testing machine for load versus deflection for the test specimens.

Possible sources of error in these measurements could include a variety of causes. First, there is the possibility for unaccounted for bending in the sample side of the balance arm that would not be measured by the potentiometer. However, estimations of this deflection based upon small displacement beam bending theory suggest that this deflection would fall in the range of 0.1 to 0.15 $\%$ of the total measured deflection and thus should not contribute significantly to any error. Another possible error source is inconsistency within the test specimen samples. This is supported by the fact that there is significant variation between the individual tests performed using the MTS machine. Third, errors in calibration of the device likely contribute significantly to the measurement error. Since the voltage readings had to be manually mapped to both linear displacement and angular position measurements, any error in that mapping would propagate through to the recorded load and deflection data. Finally, significant difficulty was found in precisely reading the potentiometer voltage. Since the total change in voltage through the test was roughly $100 \mathrm{mV}$ over a range of $6^{\circ}$ and the minimum resolution of the digital multimeter was $10 \mathrm{mV}$, this means that the measurement resolution of the sample extension was only $10 \%$ of the largest extension recorded and even worse for the lower load data points. Attempts were made to minimize this issue by recording data points at the change in value of the $10 \mathrm{mV}$ number display; however, a more desirable solution would be to use higher resolution voltage meter.

\section{Conclusions}

To summarize, a desktop version of a load/deflection measurement device based upon a balance type mechanism was proposed, designed, produced, and tested. Accuracy of the experimental data was found to be reasonable when compared against well established testing equipment. There are several possible aspects open to improvement such as the calibration of the measured parameters and the resolution of the voltage readings which would likely result in overall improved performance 
of the device. Compared with the established testing methods, this device represents a relatively low cost option for quick and easy collection of load/deflection data. The cost for all of the components and assembly is estimated to be in the range of $\$ 2,000-3,000$ compared against $\$ 20,000+$ for the established measurement equipment.

Future work on this device will likely focus on improved measurement accuracy as previously mentioned as well as improved data handling. By incorporating real time recording of the data via an interfaced computer it would be possible to plot near continuous data points rather than the discrete sampling presented here.

\section{References}

[1] J. Fiene, "P2: Oh, the stress!." https://alliance.seas.upenn.edu/ medesign/wiki/index . php/Courses/MEAM247-10C-P2, May 2011.

[2] KeyToMetals.com, "Engineering stress-strain curve." http://www.keytometals.com/ Article107.htm, May 2011.

[3] RDPElectrosence, "How it works - lvdt." http://www.rdpe.com/ex/hiw-lvdt.htm, May 2011.

[4] T. Tsugawa, "Specification drawing for model cpp-45b potentiometer," Midori America Corporation, 1994. 